\title{
Correction: Cost of cancer diagnosis using next-generation sequencing targeted gene panels in routine practice: a nationwide French study
}

Patricia Marino ${ }^{1,2} \cdot$ Rajae Touzani ${ }^{1,2} \cdot$ Lionel Perrier $^{3} \cdot$ Etienne Rouleau $^{4} \cdot$ Dede Sika Kossi $^{5} \cdot$ Zou Zhaomin $^{6}$. Nathanaël Charrier ${ }^{7} \cdot$ Nicolas Goardon $^{8}$. Claude Preudhomme ${ }^{9} \cdot$ Isabelle Durand-Zaleski $^{7} \cdot$ Isabelle Borget $^{6}$. Sandrine Baffert ${ }^{5}$ On behalf of NGSEco Group: Sandrine Baffert ${ }^{10,11} \cdot$ Emmanuel Barillot $^{10} \cdot$ Stéphane Bezieau $^{12}$. Isabelle Borget ${ }^{13}$. Lucie Coppin ${ }^{14}$. Clothilde Descapentries ${ }^{14}$. Isabelle Durand-Zaleski ${ }^{15}$. Sébastien Forget ${ }^{13}$. Thierry Frebourd ${ }^{16}$. Philippe Guardiola ${ }^{17}$ - Nicolas Goardon ${ }^{18} \cdot$ Claude Houdayer $^{10}$. Philippe Hupe ${ }^{10}$. Ludovic Lacroix $^{13}$ - Julie Leclerc ${ }^{14}$ - Alexandra Lespagnol ${ }^{19}$. Stéphanie Longuemare ${ }^{10}$ - Patricia Marino ${ }^{20}$. Jean Mosser ${ }^{19} \cdot$ Marie-Françoise Odou $^{14} \cdot$ Lionel Perrier $^{21} \cdot$ Claude Preudhomme $^{14}$. Françoise Revillion ${ }^{22}$. Etienne Rouleau ${ }^{13} \cdot$ Nicolas Sevenet $^{23} \cdot$ Isabelle Soubeyran $\cdot$ Dominique Vaur $^{18}$

Published online: 15 June 2018

(c) European Society of Human Genetics 2018

Correction to: Eur J Hum Genet. 26:314-23; https://doi.org/ 10.1038/s41431-017-0081-3; published online 24 January 2018
Since the publication of the article, it has been noted that there is an error in Table 2. Where $550 €$ is listed in the final column of the table, this should have been written as $555 €$.
Patricia Marino

patricia.marino@inserm.fr

Institut Paoli Calmettes, SESSTIM, Marseille, France

2 INSERM, IRD, SESSTIM, Sciences Economiques \& Sociales de la Santé \& Traitement de l'Information Médicale, Aix Marseille Univ, Marseille, France

3 Léon Berard Cancer Centre, GATE L-SE, UMR-CNRS 5824 Lyon, France

4 Department of Pathology and Medical Biology, Gustave Roussy, Villejuif, France

5 Rothschild Foundation, Health Economy, Paris, France

6 Gustave Roussy, Etudes et Recherche en économie de la santé, Villejuif, France

7 Hôpital Hôtel Dieu, , URC Eco Ile de France, Paris, France

8 Cancer Comprehensive Center François Baclesse, Cancer Biology and Genetics Laboratory, Caen, France

9 CHRU of Lille, Biology \& Pathology Center, Laboratory of Hematology, Lille, France
10 Comprehensive Cancer Center Curie, Paris, France

11 Rothschild Foundation, Paris, France

12 Nantes University Hospital, Nantes, France

13 Gustave Roussy, Villejuif, France

14 Lille University Hospital, Lille, France

15 Hôpital Hôtel Dieu, URC Eco Ile de France, Paris, France

16 Rouen University Hospital, Rouen, France

17 Angers University Hospital, Angers, France

18 Comprehensive Cancer Centre François Baclesse, Caen, France

19 Rennes University Hospital, Rennes, France

20 Institut Paoli-Calmettes, INSERM, IRD, SESSTIM, Aix Marseille Univ, Marseille, France

21 Léon Berard Cancer Centre, GATE L-SE UMR-CNRS 5824, Lyon, France

22 Oscar Lambret Cancer Centre, Lille, France

23 Comprehensive Cancer Center Bergonié, Bordeaux, France 


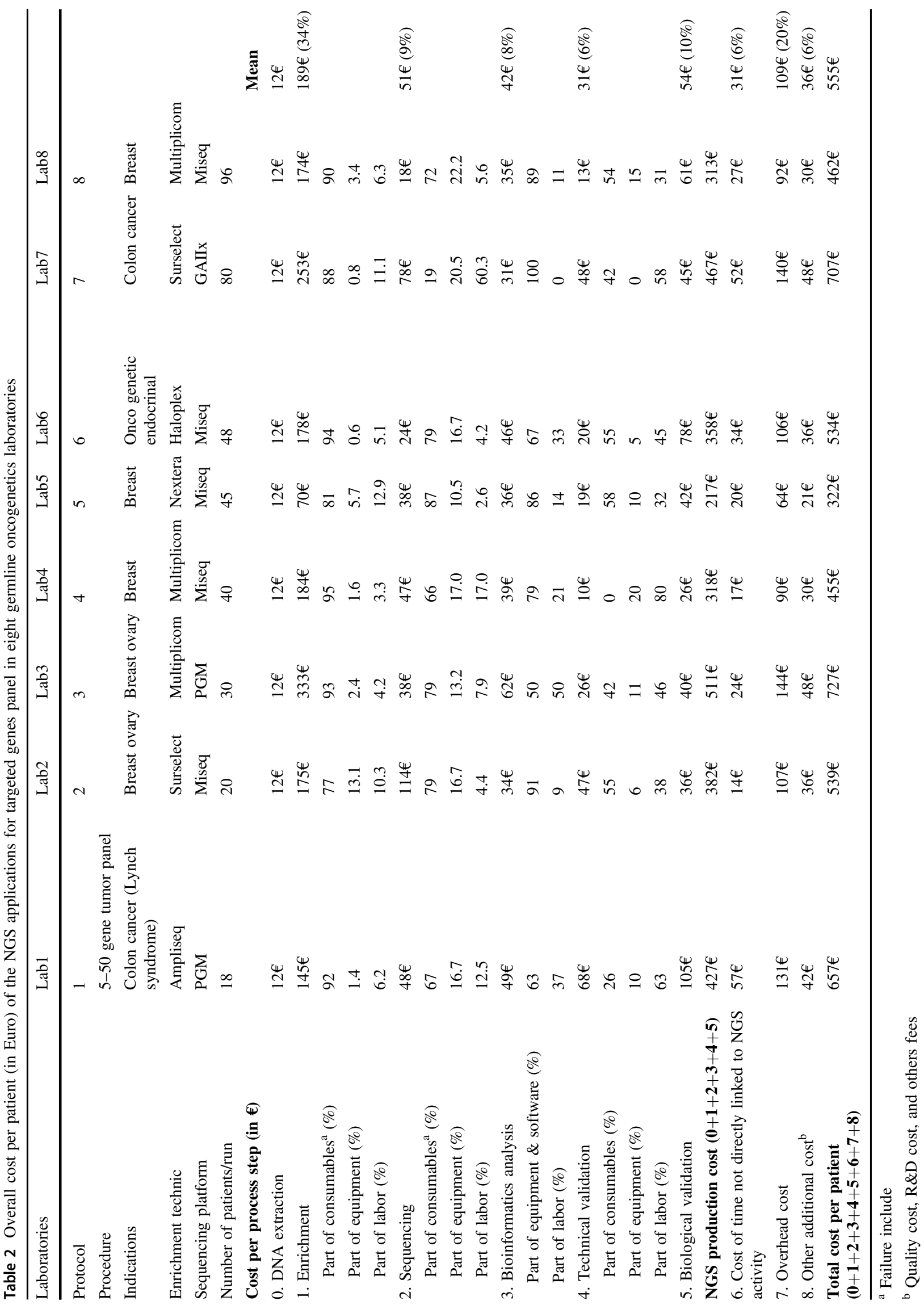

